\title{
Stochastic processes generating Schur-convex sums
}

\author{
DAWId Kotrys And Kazimierz NiKODEM(D) \\ Dedicated to Professor János Aczél on his 95th birthday.
}

\begin{abstract}
The notion of Schur-convex stochastic processes is introduced and processes generating Schur-convex sums are investigated. Some inequalities connected with convex stochastic processes are obtained. A counterpart of the result of $\mathrm{Ng}$ stating that a stochastic process generates Schur-convex sums if and only if it is Wright-convex is proved.
\end{abstract}

Mathematics Subject Classification. Primary 26A51; Secondary 39B62, 60G99.

Keywords. Convex stochastic process, Schur-convex stochastic process, Wright-convex stochastic process, Majorization, Hardy-Littlewood-Pólya theorem, Lim's inequality.

\section{Introduction}

Let $I \subset \mathbb{R}$ be an interval. Assume that $n \geqslant 2$ and $x=\left(x_{1}, \ldots, x_{n}\right), y=$ $\left(y_{1}, \ldots, y_{n}\right) \in I^{n}$. Following Schur (cf. e.g. [7,12]) we say that $x$ is majorized by $y$, and write $x \preccurlyeq y$, if there exists a doubly stochastic $n \times n$ matrix $P$ (ie. a matrix containing nonnegative elements with all rows and columns summing up to 1 ) such that $x=y \cdot P$. The notion of majorization arises in a variety of contexts. It has a deep background in economics. Issues concerning the measurment of income inequality, the distribution of wealth in a population (the Lotenz curve) or the Dalton principle of income transfer, are examples of its historical origins. Majorization arises naturally in many mathematical problems, especially in group theory and algebra. It is also useful in certain physical and chemical contexts. The terms: $x$ is more mixed (chaotic, disordered) than $y$ are directly related to majorization and can be expressed as $x \preccurlyeq y$ (see [7] for details).

A function $F: I^{n} \rightarrow \mathbb{R}$ is said to be Schur-convex if $F(x) \leqslant F(y)$ whenever $x \preccurlyeq y$, for $x, y \in I^{n}$. 
It is known, by the classical Schur theorem [12] that if a function $f: I \rightarrow \mathbb{R}$ is convex then it generates Schur-convex sums, that is the function $F: I^{n} \rightarrow \mathbb{R}$ defined by

$$
F(x)=F\left(x_{1}, \ldots, x_{n}\right)=f\left(x_{1}\right)+\cdots+f\left(x_{n}\right)
$$

is Schur-convex. It is also known that the convexity of $f$ is a sufficient but not necessary condition under which $F$ is Schur-convex. A full characterization of functions generating Schur-convex sums was given by $\mathrm{Ng}$ [8]. Namely, he proved that $F$ is Schur-convex if and only if the generating function $f$ is Wright-convex. Similar results connected with strong convexity was obtained by Nikodem, Rajba and Wąsowicz in [11] (cf. also [1]).

In this note we introduce the notion of Schur-convex stochastic processes and we present some counterparts of the results mentioned above for stochastic processes.

Let $(\Omega, \mathcal{A}, P)$ be an arbitrary probability space and $I \subset \mathbb{R}$ be an interval. A function $A: \Omega \rightarrow \mathbb{R}$ is called a random variable if it is $\mathcal{A}$-measurable. A function $X: I \times \Omega \rightarrow \mathbb{R}$ is called a stochastic process if for every $t \in I$ the function $X(t, \cdot)$ is a random variable.

Let $I \subset \mathbb{R}$ be an interval and $n \geqslant 2$. We say that a stochastic process $S: I^{n} \times \Omega \rightarrow \mathbb{R}$ is Schur-convex if

$$
x \preccurlyeq y \quad \Longrightarrow \quad S(x, \cdot) \leqslant S(y, \cdot) \quad \text { (a.e.) }
$$

for all $x, y \in I^{n}$.

Recall also that a stochastic process $X: I \times \Omega \rightarrow \mathbb{R}$ is called:

- convex if for all $x, y \in I$ and $t \in[0,1]$ the following inequality holds

$$
X(t x+(1-t) y, \cdot) \leqslant t X(x, \cdot)+(1-t) X(y, \cdot) \quad \text { (a.e.); }
$$

- Wright-convex if for all $x, y \in I$ and $t \in[0,1]$ the condition holds $X(t x+(1-t) y, \cdot)+X((1-t) x+t y, \cdot) \leqslant X(x, \cdot)+X(y, \cdot) \quad$ (a.e.);

- Jensen-convex if (1) is assumed only for $t=\frac{1}{2}$, that is for all $x, y \in I$

$$
X\left(\frac{x+y}{2}, \cdot\right) \leqslant \frac{X(x, \cdot)+X(y, \cdot)}{2} \quad \text { (a.e.). }
$$

For some properties of the above classes of processes we refer to (cf. $[2,4,5,10$, $13-15])$.

\section{Main results}

We first prove that convex stochastic processes generate Schur-convex sums.

Theorem 1. If a stochastic process $X: I \times \Omega \rightarrow \mathbb{R}$ is convex, then for every $n \geqslant 2$ the stochastic process $S_{X}: I^{n} \times \Omega \rightarrow \mathbb{R}$ given by 


$$
S_{X}\left(x_{1}, \ldots, x_{n}, \omega\right)=X\left(x_{1}, \omega\right)+\cdots+X\left(x_{n}, \omega\right), \quad\left(x_{1}, \ldots, x_{n}, \omega\right) \in I^{n} \times \Omega,
$$

is Schur-convex.

Proof. Assume that $X$ is a convex stochastic process. Let $x=\left(x_{1}, \ldots, x_{n}\right)$, $y=\left(y_{1}, \ldots, y_{n}\right)$ be elements of $I^{n}$ such that $x \preccurlyeq y$. There exists a double stochastic $n \times n$ matrix $P=\left[t_{i j}\right]$ satisfying the condition $x=y P$. Then

$$
x_{j}=\sum_{i=1}^{n} t_{i j} y_{i}, \quad j=1, \ldots, n,
$$

and by the convexity of $X$, we have

$$
\begin{aligned}
X & \left(x_{1}, \cdot\right)+\cdots+X\left(x_{n}, \cdot\right) \\
& =\sum_{j=1}^{n} X\left(x_{j}, \cdot\right)=\sum_{j=1}^{n} X\left(\sum_{i=1}^{n} t_{i j} y_{i}, \cdot\right) \\
& \leqslant \sum_{j=1}^{n} \sum_{i=1}^{n} t_{i j} X\left(y_{i}, \cdot\right)=\sum_{i=1}^{n} \sum_{j=1}^{n} t_{i j} X\left(y_{i}, \cdot\right) \\
& \left.=\sum_{i=1}^{n} X\left(y_{i}, \cdot\right) \sum_{j=1}^{n} t_{i j}=X\left(y_{1}, \cdot\right)+\cdots+X\left(y_{n}, \cdot\right) \quad \text { (a.e. }\right) .
\end{aligned}
$$

The above calculations shows that $S_{X}\left(x_{1}, \ldots, x_{n}, \cdot\right) \leqslant S_{X}\left(y_{1}, \ldots, y_{n}, \cdot\right)$, which means that $S_{X}$ is a Schur-convex stochastic process.

Many inequalities connected with convex stochastic processes can be obtained by the use of the above theorem. The corollaries below give some examples.

Corollary 2. (Jensen inequality [10]). If a stochastic process $X: I \times \Omega \rightarrow \mathbb{R}$ is convex, then for every $n \in \mathbb{N}$ and $x_{1}, \ldots, x_{n} \in I$,

$$
X\left(\frac{x_{1}+\cdots+x_{n}}{n}, \cdot\right) \leqslant \frac{X\left(x_{1}, \cdot\right)+\cdots+X\left(x_{n}, \cdot\right)}{n} \quad \text { (a.e.). }
$$

Proof. Put $\bar{x}=\frac{1}{n}\left(x_{1}+\cdots+x_{n}\right)$. Then $(\bar{x}, \ldots, \bar{x}) \preccurlyeq\left(x_{1}, \ldots, x_{n}\right)$ and hence

$$
\left.X(\bar{x}, \cdot)+\cdots+X(\bar{x}, \cdot) \leqslant X\left(x_{1}, \cdot\right)+\cdots+X\left(x_{n}, \cdot\right) \quad \text { (a.e. }\right) .
$$

The next result is a counterpart of the classical Hardy-Littlewood-Pólya majorization theorem [3] (see also $[7,9]$ ).

Corollary 3. Let $I \subset \mathbb{R}$ be an interval and $n \geqslant 2$. Assume also that $x=$ $\left(x_{1}, \ldots, x_{n}\right), y=\left(y_{1}, \ldots, y_{n}\right) \in I^{n}$ satisfy:

(a) $x_{n} \leqslant \cdots \leqslant x_{1}, y_{n} \leqslant \cdots \leqslant y_{1}$;

(b) $x_{1}+\cdots+x_{k} \leqslant y_{1}+\cdots+y_{k}, \quad k=1, \ldots, n-1$;

(c) $x_{1}+\cdots+x_{n}=y_{1}+\cdots+y_{n}$. 
If a stochastic process $X: I \times \Omega \rightarrow \mathbb{R}$ is convex, then

$$
X\left(x_{1}, \cdot\right)+\cdots+X\left(x_{n}, \cdot\right) \leqslant X\left(y_{1}, \cdot\right)+\cdots+X\left(y_{n}, \cdot\right) \quad \text { (a.e.). }
$$

Proof. Note that assumptions (a)-(c) imply $x \preccurlyeq y$ (see e.g. [7]) and apply Theorem 1.

By Corollary 3 we get a counterpart of the Lim inequality (cf. [6]) for convex stochastic processes.

Corollary 4. Let $a \geqslant 0, b \geqslant 0, c \geqslant a+b$ be real numbers, and let $X:[0,+\infty) \times$ $\Omega \rightarrow \mathbb{R}$ be a convex stochastic process. Then

$$
X(a, \cdot)+X(b+c, \cdot) \geqslant X(a+b, \cdot)+X(c, \cdot) \quad \text { (a.e.). }
$$

Proof. We use Corollary 3 for $n=2, x_{1}=c, x_{2}=a+b, y_{1}=b+c, y_{2}=a$, and the inequality (4) follows.

The next result is connected with the Shannon information entropy.

Corollary 5. Let $Y: \Omega \rightarrow \mathbb{R}$ be a random variable and $H:(0, \infty)^{n} \times \Omega \rightarrow \mathbb{R}$ be a stochastic process defined by

$$
H\left(p_{1}, \ldots, p_{n}, \omega\right)=-\left(p_{1} \ln p_{1}+\cdots+p_{n} \ln p_{n}\right) Y(\omega) .
$$

Then, for every $p_{1}, \ldots, p_{n}>0$ such that $p_{1}+\cdots+p_{n}=1$, we have

$$
H\left(\frac{1}{n}, \ldots, \frac{1}{n}, \cdot\right) \geqslant H\left(p_{1}, \ldots, p_{n}, \cdot\right) \geqslant H(1,0, \ldots, 0, \cdot) \quad \text { (a.e.). }
$$

Proof. The stochastic process $X:(0, \infty) \times \Omega \rightarrow \mathbb{R}$ defined by $X(p, \omega)=$ $(p \ln p) Y(\omega)$ is convex. Since for every $p_{1}, \ldots, p_{n}>0$ such that $p_{1}+\cdots+p_{n}=1$

$$
\left(\frac{1}{n}, \ldots, \frac{1}{n}\right) \preccurlyeq\left(p_{1}, \ldots, p_{n}\right) \preccurlyeq(1,0, \ldots, 0),
$$

we get (5) by Theorem 1 .

We will show now that processes generating Schur-convex sums must be Jensen-convex.

Theorem 6. If for some $n \geqslant 2$ the process $S_{X}: I^{n} \times \Omega \rightarrow \mathbb{R}$ given by

$$
S_{X}\left(x_{1}, \ldots, x_{n}, \omega\right)=X\left(x_{1}, \omega\right)+\cdots+X\left(x_{n}, \omega\right), \quad\left(x_{1}, \ldots, x_{n}, \omega\right) \in I^{n} \times \Omega,
$$

is Schur-convex, then the process $X: I \times \Omega \rightarrow \mathbb{R}$ is Jensen-convex.

Proof. Take $y_{1}, y_{2} \in I$ and put $x_{1}=x_{2}=\frac{1}{2}\left(y_{1}+y_{2}\right)$. Let us consider the points $y=\left(y_{1}, y_{2}, y_{2}, \ldots, y_{2}\right)$ and $x=\left(x_{1}, x_{2}, y_{2}, \ldots, y_{2}\right)$ (if $n=2$, we take $\left.y=\left(y_{1}, y_{2}\right), x=\left(x_{1}, x_{2}\right)\right)$. Then $x=y \cdot P$ with

$$
P=\left[\begin{array}{ccccc}
\frac{1}{2} & \frac{1}{2} & 0 & \ldots & 0 \\
\frac{1}{2} & \frac{1}{2} & 0 & \ldots & 0 \\
0 & 0 & 1 & \ldots & 0 \\
\vdots & \vdots & \vdots & \ddots & \vdots \\
0 & 0 & 0 & \ldots & 1
\end{array}\right]
$$


which means that $x \preccurlyeq y$. Therefore, by the Schur-convexity of $S_{X}$, we obtain

$$
S_{X}\left(x_{1}, x_{2}, y_{2}, \ldots, y_{2}, \cdot\right) \leqslant S_{X}\left(y_{1}, y_{2}, y_{2}, \ldots, y_{2}, \cdot\right) \quad \text { (a.e.), }
$$

and finally

$$
\left.2 X\left(\frac{y_{1}+y_{2}}{2}, \cdot\right) \leqslant X\left(y_{1}, \cdot\right)+X\left(y_{2}, \cdot\right) \quad \text { (a.e. }\right) \text {. }
$$

This shows that $X$ is Jensen-convex, which was to be proved.

As was shown above in Theorems 1 and 6 , if a stochastic process $X$ : $I \times \Omega \rightarrow \mathbb{R}$ is convex, then for every $n \geqslant 2$ the corresponding process $S_{X}$ is Schur-convex and if for some $n \geqslant 2$ the process $S_{X}$ is Schur-convex, then $X$ is Jensen-convex. The next theorem characterizes all the processes $X$ for which $S_{X}$ are Schur-convex. It is a counterpart of the result of $\mathrm{Ng}$ [8] on functions generating Schur-convex sums.

Theorem 7. The following conditions are equivalent:

(i) For every $n \geqslant 2$ the stochastic process $S_{X}: I^{n} \times \Omega \rightarrow \mathbb{R}$ defined by

$$
S_{X}\left(x_{1}, \ldots, x_{n}, \omega\right)=X\left(x_{1}, \omega\right)+\ldots+X\left(x_{n}, \omega\right), \quad\left(x_{1}, \ldots, x_{n}, \omega\right) \in I^{n} \times \Omega,
$$

is Schur-convex.

(ii) For some $n \geqslant 2$ the stochastic process $S_{X}$ given by (6) is Schur-convex.

(iii) The stochastic process $X: I \times \Omega \rightarrow \mathbb{R}$ is Wright-convex.

(iv) There exist a convex stochastic process $Y: I \times \Omega \rightarrow \mathbb{R}$ and an additive stochastic process $A: I \times \Omega \rightarrow \mathbb{R}$ such that

$$
X(x, \cdot)=Y(x, \cdot)+A(x, \cdot) \quad \text { (a.e.), } \quad x \in I .
$$

Proof. The implication $(i) \Rightarrow(i i)$ is obvious.

To see that $(i i) \Rightarrow($ iii $)$ holds fix $y_{1}, y_{2} \in I, t \in(0,1)$. We put $x_{1}=t y_{1}+(1-$ $t) y_{2}, x_{2}=(1-t) y_{1}+t y_{2}$ and, if $n>2$, take additionally $x_{i}=y_{i}=z \in I$ for $i=3, \ldots, n$. Then, by a similar argumentation as in the proof of Theorem 6 we get

$$
\left(x_{1}, \ldots, x_{n}\right) \preccurlyeq\left(y_{1}, \ldots, y_{n}\right) .
$$

By the Schur-convexity of $S_{X}$ we have

$$
\left.S_{X}\left(x_{1}, \ldots, x_{n}, \cdot\right) \leqslant S_{X}\left(y_{1}, \ldots, y_{n}, \cdot\right) \quad \text { (a.e. }\right) .
$$

Therefore

$$
\left.X\left(t y_{1}+(1-t) y_{2}, \cdot\right)+X\left((1-t) y_{1}+t y_{2}, \cdot\right) \leqslant X\left(y_{1}, \cdot\right)+X\left(y_{2}, \cdot\right) \quad \text { (a.e. }\right),
$$

which shows that the process $X$ is Wright-convex.

The implication (iii) $\Rightarrow(i v)$ follows from Skowroński's theorem giving the representation of Wright-convex stochastic processes (cf. [15], Theorem on page $31)$. 
To prove $(i v) \Rightarrow(i)$ assume that $X$ has the representation of the form (7). By the convexity of $Y$ and by Theorem 1 the process $Y$ generates Schur-convex sums $S_{Y}$, that is, for any $x=\left(x_{1}, \ldots, x_{n}\right) \preccurlyeq y=\left(y_{1}, \ldots, y_{n}\right)$, the following condition holds

$$
Y\left(x_{1}, \cdot\right)+\cdots+Y\left(x_{n}, \cdot\right) \leqslant Y\left(y_{1}, \cdot\right)+\cdots+Y\left(y_{n}, \cdot\right) \quad \text { (a.e.). }
$$

Note also that by the additivity of $A$, similarly as in the proof of Theorem 1 , we get

$$
\begin{aligned}
A\left(x_{1}, \cdot\right)+\cdots+A\left(x_{n}, \cdot\right) & =A\left(x_{1}+\cdots+x_{n}, \cdot\right) \\
& \left.=A\left(y_{1}+\cdots+y_{n}, \cdot\right)=A\left(y_{1}, \cdot\right)+\cdots+A\left(y_{n}, \cdot\right) \quad \text { (a.e. }\right) .
\end{aligned}
$$

Using the above inequalities we obtain

$$
\begin{aligned}
X\left(x_{1}, \cdot\right)+\cdots+X\left(x_{n}, \cdot\right) & =Y\left(x_{1}, \cdot\right)+\cdots+Y\left(x_{n}, \cdot\right)+A\left(x_{1}, \cdot\right)+\cdots+A\left(x_{n}, \cdot\right) \\
& \leqslant Y\left(y_{1}, \cdot\right)+\cdots+Y\left(y_{n}, \cdot\right)+A\left(y_{1}, \cdot\right)+\cdots+A\left(y_{n}, \cdot\right) \\
& \left.=X\left(y_{1}, \cdot\right)+\cdots+X\left(y_{n}, \cdot\right) \quad \text { (a.e. }\right),
\end{aligned}
$$

which shows that $S_{X}$ is Schur-convex. This finishes the proof.

Open Access. This article is distributed under the terms of the Creative Commons Attribution 4.0 International License (http://creativecommons.org/licenses/by/4.0/), which permits unrestricted use, distribution, and reproduction in any medium, provided you give appropriate credit to the original author(s) and the source, provide a link to the Creative Commons license, and indicate if changes were made.

Publisher's Note Springer Nature remains neutral with regard to jurisdictional claims in published maps and institutional affiliations.

Funding Funding was provided by University of Bielsko-Biala (KM/1/2019).

\section{References}

[1] Dragomir, S.S., Nikodem, K.: Functions generating $(m, M, \Psi)$-Schur-convex sums. Aequat. Math. https://doi.org/10.1007/s00010-018-0569-0. (in press)

[2] González, L., Kotrys, D., Nikodem, K.: Separation by convex and strongly convex stochastic processes. Publ. Math. Debr. 89(3), 365-372 (2016)

[3] Hardy, G.H., Littlewood, J.E., Pólya, G.: Inequalities. Cambridge University Press, Cambridge (1952)

[4] Kotrys, D.: Hermite-Hadamard inequality for convex stochastic processes. Aequat. Math. 83, 143-151 (2012)

[5] Kotrys, D.: Remarks on strongly convex stochastic processes. Aequat. Math. 86, 91-98 (2012)

[6] Kuczma, M.: An Introduction to the Theory of Functional Equations and Inequalities. Cauchy's Equation and Jensen's Inequality, PWN-Uniwersytet Śląski, WarszawaKraków-Katowice, 1985. 2nd edn. Birkhäuser, Basel, Boston, Berlin (2009)

[7] Marshall, A.W., Olkin, I., Arnold, B.C.: Inequalities: Theory of Majorization and Its Applications. Springer, New York (2011) 
[8] Ng, C.T.: Functions generating Schur-convex sums. In: General Inequalities 5 (Oberwolfach, 1986), International Series of Numerical Mathematics, vol. 80, pp. 433-438. Birkhäuser Verlag, Basel (1987)

[9] Niculescu, C.P., Rovenéa, I.: Hardy-Littlewood-Polya theorem of majorization in the framework of generalized convexity. Carpath. J. Math. 33(1), 87-95 (2017)

[10] Nikodem, K.: On convex stochastic processes. Aequat. Math. 20, 184-197 (1980)

[11] Nikodem, K., Rajba, T., Wa̧sowicz, S.: Functions generating strongly Schur-convex sums. In: Bandle, C., et al. (eds.) Inequalities and Applications 2010. International Series of Numerical Mathematics, vol. 161, pp. 175-182. Springer, Basel (2012)

[12] Schur, I.: Über eine Klasse von Mittelbildungen mit Anwendungen auf die Determinantentheorie. Sitzungsber. Berl. Math. Ges. 22, 9-20 (1923)

[13] Shaked, M., Shanthikumar, J.G.: Stochastic convexity and its applications. Adv. Appl. Prob. 20, 427-446 (1988)

[14] Skowroński, A.: On some properties of J-convex stochastic processes. Aequat. Math. 44, 249-258 (1992)

[15] Skowroński, A.: On Wright-convex stochastic processes. Ann. Math. Sil. 9, 29-32 (1995)

Dawid Kotrys and Kazimierz Nikodem

Department of Mathematics

University of Bielsko-Biala

ul. Willowa 2

43-309 Bielsko-Biała

Poland

e-mail: dkotrys@ath.bielsko.pl

Kazimierz Nikodem

e-mail: knikodem@ath.bielsko.pl

Received: February 19, 2019

Revised: May 13, 2019 